\title{
The Effect of Nrf2 Knockout on Ocular Surface Protection from Acute Tobacco Smoke Exposure
}

\section{Evidence from Nrf2 Knockout Mice}

\author{
Takashi Kojima, ${ }^{*}$ Murat Dogru, ${ }^{* \dagger}$ Akihiro Higuchi, ${ }^{*}$ Taeko Nagata, ${ }^{*}$ Osama M.A. Ibrahim, ${ }^{*}$ Takaaki Inaba, and Kazuo Tsubota*
}

From the Department of Ophthalmology, ${ }^{*}$ Keio University School of Medicine, Shinjuku-ku, Tokyo; and the Department of Ophthalmology, ${ }^{\dagger}$ Tokyo Dental College, Ichikawa, Japan

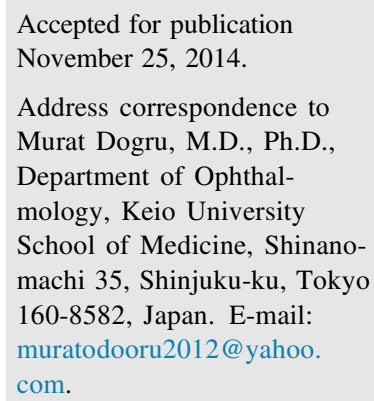

\begin{abstract}
Ocular surface mucosa is the first-line ocular tissue to be exposed to environmental stress. We evaluated tear functions and keratoconjunctival epithelial alterations after sidestream cigarette smoke (SCS) exposure and tried to clarify the role of the transcription factor nuclear factor erythroid 2-related factor 2 (Nfe2l2, also known as Nrf2), on the ocular surface. In wild-type and $\mathrm{Nrf2^{-/- }}$ mice, tear volume did not change after SCS exposure. Tear film breakup time (tear stability) in $\mathrm{Nrf}^{-/-}$mice was significantly shorter than that in wildtype mice after SCS exposure. Vital staining scores, including fluorescein and Rose Bengal staining, showed significantly higher values in $\mathrm{Nrf2}^{-/-}$mice than in wild-type mice after SCS exposure. Excessive oxidative stress accumulation was detected in $\mathrm{Nrf2}^{-/-}$mice after SCS exposure using immunohistochemical analysis. Immunohistochemical analysis also revealed decreased mucin 1 (Muc1) and Muc5ac staining in $\mathrm{Nrf}^{-/-}$mice after SCS exposure. mRNA expression levels of Muc1, Muc4, and Muc5ac and of SAM-pointed domain epithelial-specific transcription factor in $\mathrm{Nrf2}^{-/-}$mice were lower than those in wild-type mice after SCS exposure. Mean tear IL-6 concentrations increased significantly in $\mathrm{Nrf2}^{-/-}$mice after SCS exposure. In conclusion, SCS exposure induced decreased tear stability, ocular surface damage, and altered conjunctival phenotype in $\mathrm{Nrf2}^{-/-}$mice. Nrf2 could play an important role in protection of the ocular surface against SCS exposure. (Am J Pathol 2015, 185: 776-785; http://dx.doi.org/10.1016/j.ajpath.2014.11.014)
\end{abstract}

There are 4000 or more active substances in tobacco smoke, and most are hazardous to human health, with cytotoxic effects to the pulmonary and cardiovascular systems. ${ }^{1}$ Cigarette smoke contains ammonia, carbon monoxide, formaldehyde, benzene, nicotine, various gases and particles, and an assortment of potentially genotoxic and/or carcinogenic organic compounds. ${ }^{2}$ Mice after cigarette smoke exposure developed basal cell hyperplasia and squamous metaplasia of the nasal cavity and hypertrophy of Clara cells. ${ }^{3}$

Long-term active cigarette smoking has been reported to induce qualitative and quantitative changes in the tear film and the ocular surface, resulting in dry eyes. ${ }^{4}$ Demographic surveys have shown that $25 \%$ to $40 \%$ of employees experience cigarette smoke in their work environment and that $25 \%$ of them have dry eye-related symptoms.

According to the Beaver Dam Eye Study, a relation between current active smoking/history of smoking and onset of dry eye disease was reported in the elderly. ${ }^{6}$ The Blue Mountains Eye Study also pointed out that smoking was one of the important risk factors for dry eyes in a similar population. ${ }^{7}$

In a recent study, the deleterious effects of brief passive tobacco smoke exposure on the tear film and the ocular surface were confirmed. ${ }^{8}$ Herein, increased tear inflammatory cytokine levels and lipid peroxidation products and decreased mucosal defenses were reported to result in tear instability and damage to the ocular surface epithelia. This study also showed that even brief passive cigarette smoke exposure induced increased tear evaporation, oxidation of tear/ocular surface lipids, decreased tear film lipid spread time, increased IL-6 concentrations in tears, and mucin 5ac (Muc5ac) mRNA expression.

Supported by research grant FP01210115 from the Smoking Research Foundation.

Disclosures: None declared. 
Recently, we established a smoking rat model and reported that the ocular surface was damaged after mainstream cigarette smoke exposure, probably through DNA oxidation by reactive oxygen species produced by cytochrome P450s. ${ }^{9}$

Several studies revealed that brief acute smoke exposure on the ocular surface could alter tear functions and damage the ocular surface epithelium owing to the accumulation of reactive oxygen species or increased inflammatory responses. ${ }^{9,10}$ However, the upstream mechanism of ocular surface protection against tobacco smoke exposure was not well-known.

Nuclear factor erythroid 2-related factor 2 (Nfe212, also known as Nrf2) is a transcription factor that regulates downstream target genes encoding biological defense enzymes, including antioxidant and detoxification enzymes, to protect the tissues. ${ }^{11}$ We used $\mathrm{Nrf}^{2}{ }^{-/-}$mice and investigated the effect of passive sidestream cigarette smoke (SCS) exposure on the ocular surface and tear functions.

The present study was designed to investigate the upstream mechanism of ocular surface damage after SCS exposure. We compared tear functions and histopathologic findings between wild-type (WT) mice and $\mathrm{Nrf} 2^{-/-}$mice to investigate the involvement of Nrf2 in the pathogenesis of ocular surface damage after cigarette smoke exposure.

\section{Materials and Methods}

Animals

Twelve Nrf2 $2^{-1-}$ male mice with C57BL/background and 12 C57BL/6 strain WT male mice were examined at 10 weeks with or without cigarette smoke exposure. $\mathrm{Nrf}^{-/-}$mice were received from the Department of Medical Biochemistry at Tohoku University (Sendai, Japan), and the WT C57BL/6 mice were purchased from Japan Clea (Osaka, Japan). Generation of $N r f 2^{-1-}$ mice was previously described elsewhere. ${ }^{12}$ All the studies were performed in accordance with the Association for Research in Vision and Ophthalmology Statement for the Use of Animals in Ophthalmic and Visual Research.

\section{Exposure to SCS}

Smoking treatment was performed by exposing the mice to SCS. Briefly, the mice were placed in an experimental smoking chamber $(60 \times 40 \times 35 \mathrm{~cm})$ for 3 hours with continuous fresh air ventilation (15 mL per second). A lit cigarette containing $14 \mathrm{mg}$ of tar (Seven Stars; Japan Tobacco, Tokyo, Japan) was placed into the smoking chamber every 30 minutes for a total of six times in 3 hours for 5 days during the exposure period. All the SCS exposure experiments started at $1 \mathrm{PM}$. The air conditions of the smoking room in this study were comparable with those of a smoking room for humans. The carbon monoxide concentration in the smoking chamber was monitored during the smoking experiment. Because the average and maximum concentrations of carbon monoxide in the smoking chamber were almost 100 and $250 \mathrm{ppm}$, respectively, the toxicologic effects of carbon monoxide on the mice were not considered in this smoking experiment.

\section{Aqueous Tear Production Measurements}

Aqueous tear production was measured using phenol red-impregnated cotton threads (Zone-Quick; Showa Yakuhin Kako Co. Ltd., Tokyo, Japan) without anesthesia. The validity of this test in mice has been described in previous studies. ${ }^{13}$ The threads were held using jeweler's forceps and then immersed into the tear meniscus in the lateral canthus for 60 seconds. The length of wetting of the thread was measured in millimeters. Aqueous tear production was weight adjusted by dividing the amount of total aqueous tear produced in 60 seconds by weight.

\section{Ocular Surface Epithelial Damage Assessment}

Corneal fluorescein staining was evaluated using slit-lamp biomicroscopy and cobalt blue light after instillation of $2 \mu \mathrm{L}$ of $0.5 \%$ sodium fluorescein. Excess fluorescein was wiped from the lateral tear meniscus. The cornea was examined using a handheld slit lamp 2 minutes after fluorescein instillation. Punctuate staining was recorded using a grading system of 0 to 3 points for the superior, central, and inferior corneal areas. Fluorescein staining scores ranged from a minimum of 0 to a maximum of 9 points. Similarly, $1 \%$ Rose Bengal staining was performed and scored in the same manner.

\section{Measurement of Tear Film Breakup Time}

After instillation of $1 \mu \mathrm{L}$ of $0.5 \%$ sodium fluorescein, excess fluorescein was wiped away. After spontaneous blinking, the time until the appearance of a dark area representing tear film breakup was measured three times, and the averaged value was used for analysis.

Corneal and Conjunctival Specimen Collections for Immunohistochemical Analysis and Real-time RT-PCR

Animals were sacrificed before and after SCS exposure. Eyes were enucleated, including eyelids and palpebral conjunctiva. After enucleation, the conjunctiva was trimmed from the eyeball, and then the cornea was obtained with fine scissors. For real-time RT-PCR experiments, tissues were immediately immersed into RNAlater (Applied Biosystems, Carlsbad, CA). For immunohistochemical analysis, enucleated eyeballs were directly frozen in optimum cutting temperature compound.

Immunohistochemical Analysis Staining for 0xidative Stress Markers

Lipid peroxidation was assessed by immunohistochemical detection of 4-hydroxy-2-nonenal (4-HNE). Oxidative DNA damage was investigated by immunohistochemical staining 

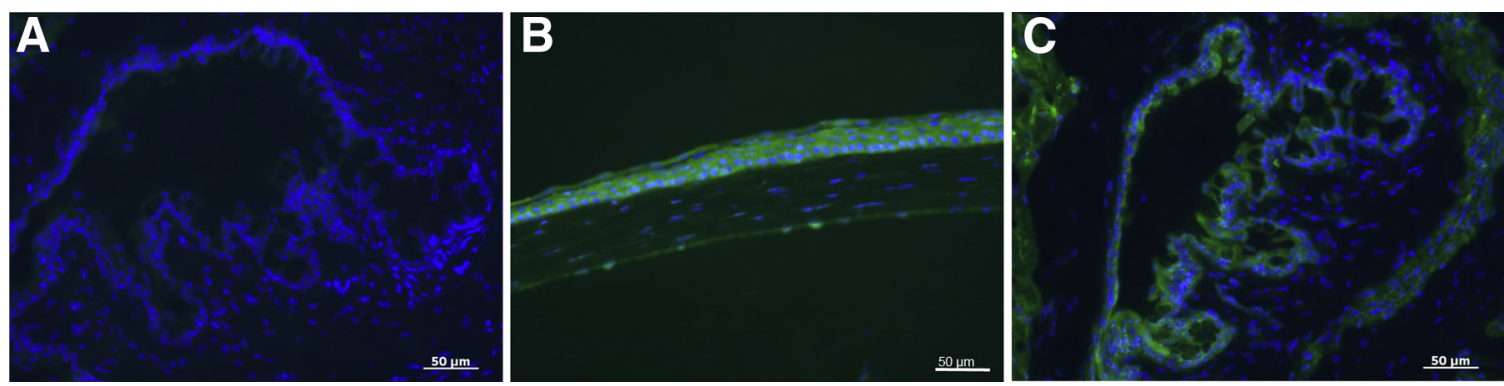

Figure 1 Localization of nuclear factor erythroid 2-related factor 2 (Nrf2) in conjunctival and corneal tissues. A: Representative immunohistochemical staining for Nrf2 using isotype control showed scanty staining in conjunctival tissue. B: Nrf2 was highly expressed in the full layer of corneal epithelial cells and endothelial cells. C: In conjunctival tissue, Nrf2 also localized in the full layer of conjunctival epithelium.

with anti-8-hydroxy-2'-deoxyguanosine (8-OHdG) antibodies. The avidin-biotin-peroxidase complex method was used in immunostaining. Briefly, 6- $\mu \mathrm{m}$ cryosections from mouse corneal/conjunctival tissue were fixed in $4 \%$ paraformaldehyde for 20 minutes. To block nonspecific background staining, tissue sections were treated with normal horse serum (Vector Laboratories, Burlingame, CA) for 2 hours at room temperature. The tissues were then treated with mouse anti-8-OHdG monoclonal antibody (Japan Institute for the Control of Aging, Shizuoka, Japan) at a concentration of 10 $\mu \mathrm{g} / \mathrm{mL}$ diluted with horse blocking serum and with anti4-HNE monoclonal antibody (Japan Institute for the Control of Aging) at a concentration of $25 \mu \mathrm{g} / \mathrm{mL}$ diluted with horse blocking serum for 2 hours at room temperature. For negative controls, the primary antibody was replaced with mouse $\mathrm{IgG} 1$ isotype control (MOPC-21; Sigma-Aldrich, St. Louis, MO). Endogenous peroxidase activity was blocked using $3 \% \mathrm{H}_{2} \mathrm{O}_{2}$ in methanol for 3 minutes. The sections were incubated for 30 minutes with biotin-labeled horse anti-mouse $\operatorname{IgG}$ serum (Vector Laboratories), followed by avidin-biotin-alkaline phosphatase complex treatment (Vector Laboratories) for 30 minutes. The sections were washed in phosphate-buffered saline, developed in diaminobenzidine chromogen solution (Vector Laboratories), lightly counterstained with hematoxylin for 4 minutes at room temperature, washed with tap water, dehydrated, and mounted. Endogenous peroxidase activity was blocked using 3.0\% $\mathrm{H}_{2} \mathrm{O}_{2}$ in methanol for 3 minutes. The sections were incubated for 30 minutes with biotin-labeled rabbit anti-rat IgG serum (Vector Laboratories), followed by avidin-biotin-alkaline phosphatase complex treatment (Vector Laboratories) for 30 minutes. The sections were washed in 0.1 $\mathrm{mol} / \mathrm{L}$ phosphate-buffered saline, developed in prepared diaminobenzidine chromogen solution (Vector Laboratories), lightly counterstained with hematoxylin for 4 minutes at room temperature, washed with tap water, dehydrated, and mounted.

\section{Fluorescent Immunohistochemical Staining for Nrf2,} Muc1, and Muc5ac in the Ocular Surface

To evaluate the localization and expression levels of Nrf2, Muc1, and Muc5ac in the ocular surface, fluorescent immunohistochemical analysis was performed as follows. Briefly, $6-\mu \mathrm{m}$ cryosections from the mouse eyeball were fixed in $4 \%$ paraformaldehyde for 20 minutes. After blocking with $1 \%$ bovine serum albumin in phosphate-buffered saline containing $2 \%$ donkey serum, sections were incubated overnight with primary antibodies. After washing with phosphate-buffered saline, the sections were incubated for 30 minutes with secondary antibodies and were observed using a fluorescence microscope (Carl Zeiss Inc., Oberkochen, Germany). For negative controls, isotype control IgG was applied instead of primary antibody. The specimens were immunostained using the following primary antibodies: rabbit anti-Nrf2 antibody (0.01 mg/mL, ab31163; Abcam, Boston, MA), mouse antiMuc5ac antibody $(2 \mu \mathrm{g} / \mathrm{mL}$, MS-145-P0; Thermo Fisher Scientific Inc., Cheshire, UK), and rabbit anti-Muc1 (0.2 mg/ $\mathrm{mL}$, ab8878; Abcam). The secondary antibody was fluorescein isothiocyanate-conjugated anti-rabbit IgG antibody $(0.0075 \mathrm{mg} / \mathrm{mL}$; Jackson ImmunoResearch Laboratories, West Grove, PA). DAPI (Vector Laboratories) was used for nuclear staining.

\section{Quantitative RT-PCR for Ocular Surface Mucins and Spdef}

Mouse cornea and conjunctival tissues were preserved overnight in RNAlater (Applied Biosystems) after prompt excision. Tissues were then transferred into Isogen reagent (Nippon Gene Co., Tokyo, Japan) and homogenized well. Total RNA was extracted, cleaned up, and treated with DNase using the RNeasy mini kit (Qiagen Inc., Valencia, CA). cDNA synthesis was performed using the iScript cDNA synthesis kit (Bio-Rad Laboratories, Hercules, CA). SYBR Green-based quantitative real-time PCR was performed using the StepOnePlus system (Applied Biosystems). Mouse glyceraldehyde3-phosphate dehydrogenase (sense: $5^{\prime}$-TGACGTGCCGCCTGGAGAAA-3', antisense: 5'-GACTTCCCGTAGAACCCGATGTGA-3'), Muc1 (sense: $5^{\prime}$-CTTTCAGAAGACTCCGCCAG-3', antisense: 5'-CGAGACTTAGTCAGAACCGG-3'), Muc4 (sense: $5^{\prime}$-CTCCAAGAAATGTAGTGGCTTTCAG-3', antisense: 5'-ATGAGGTCGGGTTCTGGCAC-3'), Muc5ac (sense: 5'-AAAGACACCAGTAGTCACTCAGCAA-3', antisense: $5^{\prime}$-ACCAAACTGTGACTGAAGGGTC- $3^{\prime}$ ), and SAMpointed domain epithelial-specific transcription factor (Spdef; sense: 5'-TTGGATGAGCACTCGCTAGA-3', antisense: $5^{\prime}$-TTGCACGTCTTCACCGAAAA-3') primers were used 

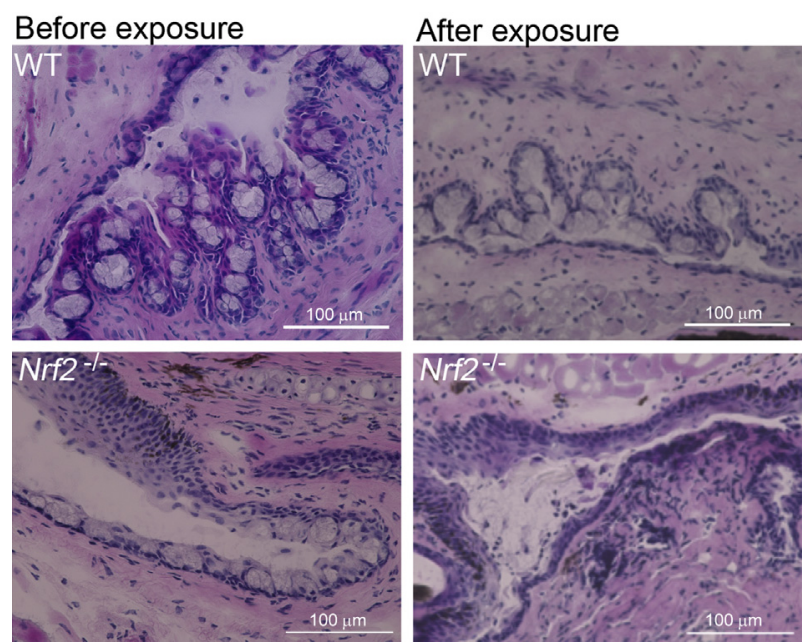

Figure 2 Representative hematoxylin and eosin staining samples before and after sidestream cigarette smoke (SCS) exposure in wild-type (WT) and $\mathrm{Nrf2}{ }^{-/}$mice. WT mice showed normal architecture of the conjunctival epithelium with abundant goblet cells and lacked any changes before and after SCS exposure. Before SCS exposure, $\mathrm{Nrf2}^{-/-}$mice had normal architecture of the conjunctival epithelium with goblet cells except partial thickening of the conjunctival epithelium. After SCS exposure in $\mathrm{Nrf2}^{-/-}$ mice, marked subconjunctival cellular infiltration, hyperplasia of the conjunctival epithelium, and decreased goblet cells were observed.

as templates. Data were normalized to glyceraldehyde-3phosphate dehydrogenase.

\section{Cytometric Bead Array for Assessment of Inflammatory Cytokines in Tears}

The BD cytometric bead array system (BD Biosciences, San Jose, CA) was used to investigate the sensitivity of amplified fluorescence detection by flow cytometry to measure soluble analyses in particle-based immunoassay. Each bead provides a capture surface for a specific protein and is analogous to an individual coated well in an enzyme-linked immunosorbent assay plate. The testing allows the detection of multiple analyses in a small volume sample. We quantitatively measured IL-1, IL-2, IL-6, IL-10, interferon- $\gamma$, and tumor necrosis factor protein levels in tear samples using the BD mouse inflammation kit (BD Biosciences).

A tear sample was collected after instilling $10 \mu \mathrm{L}$ of saline twice and was centrifuged at $10,000 \mathrm{rpm}$ for 5 minutes at $4^{\circ} \mathrm{C}$. After reconstituting the mouse inflammation standards, $20 \mu \mathrm{L}$ of a cytokine standard mixture and $20-\mu \mathrm{L}$ tear samples were diluted with $30 \mu \mathrm{L}$ of the assay diluent. Each standard and sample was added to a mixture of $50 \mu \mathrm{L}$ of capture Ab-bead reagent and detector Ab-PE reagent. The mixture $(150 \mu \mathrm{L})$ was subsequently incubated for 2 hours at room temperature and was washed with $1 \mathrm{~mL}$ of wash buffer (from the kit) to remove unbound detector $\mathrm{Ab}-\mathrm{PE}$ reagent. After washing, the samples and standards were centrifuged at $200 \times g$ for 5 minutes, and then the supernatants were carefully removed. The bead pellets were resuspended with $300 \mu \mathrm{L}$ of wash buffer before data acquisition using flow cytometry. Flow cytometric analysis was performed using a FACSCalibur flow cytometer
(BD Immunocytometry Systems, San Jose, CA). Data were acquired and analyzed using the BD cytometric bead array software version 1.4 (BD Biosciences).

\section{Results}

Immunohistochemical Expression of Nrf2 in the Ocular Surface Epithelium

Nrf2 was highly expressed in all layers of the corneal and conjunctival epithelia and the corneal endothelium (Figure 1).

Conjunctival Hyperplasia and Marked Subconjunctival Cellular Infiltration Are the Prominent Alterations in $\mathrm{Nrf2}^{-/-}$Mice after SCS Exposure

Hematoxylin and eosin staining was performed to evaluate the histopathologic changes after SCS exposure. Although significant differences between WT and $\mathrm{Nrf}^{-/-}$mice were not observed before SCS exposure, thickening of the conjunctival epithelium and marked cellular infiltration under the conjunctival epithelium in $\mathrm{Nrf}^{-/-}$mice was observed after SCS exposure (Figure 2).

\section{Decrease in Tear Film Stability after SCS Exposure in $\mathrm{Nrf2}^{-/-}$Mice}

Although there were no differences in mean tear film breakup times between WT and $\mathrm{Nr}^{-/-}$mice before SCS exposure, mean breakup times in $\mathrm{Nrf}^{-1-}$ mice were significantly shorter than those in WT mice after SCS exposure (Figure 3A). There were no significant differences in tear quantity between WT and $\mathrm{Nrf}^{-/-}$mice before and after SCS exposure (Figure 3B).

\section{Deterioration of Ocular Vital Staining after SCS Exposure in $\mathrm{Nrf2}^{-/-}$Mice}

There was no significant difference in mean fluorescein staining scores before versus after SCS exposure in WT
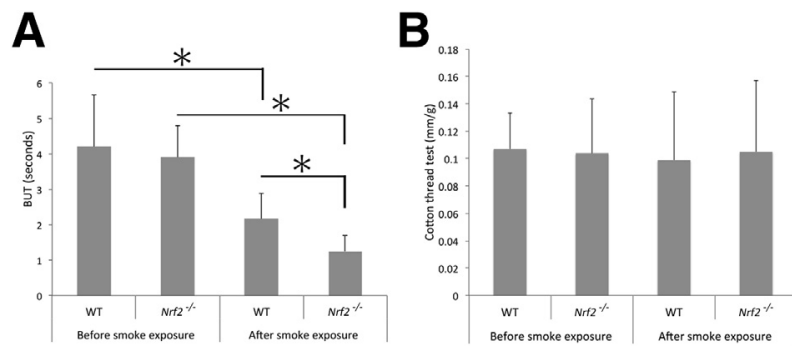

Figure 3 Tear functions before and after sidestream cigarette smoke (SCS) exposure. A: Tear film breakup time (BUT) showed significant decreases in wild-type (WT) and $\mathrm{Nrf2}^{-/-}$mice after SCS exposure. BUTs after SCS exposure in $\mathrm{Nrf2}^{-1-}$ mice were significantly shorter than those in WT mice. B: Tear volume did not show any differences before versus after SCS exposure in WT and $\mathrm{Nrf2}^{-/-}$mice. Data are given as means \pm SD. ${ }^{*} P<0.05$. 


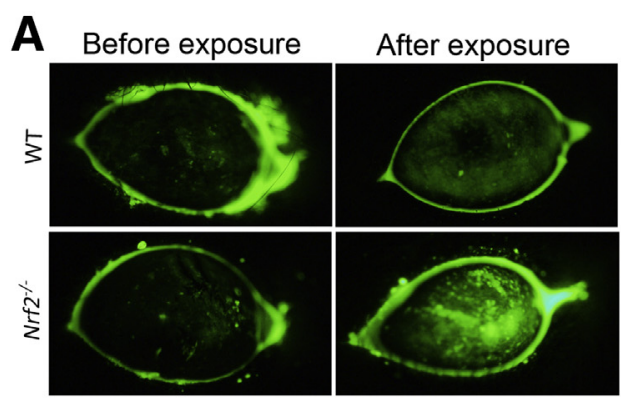

C

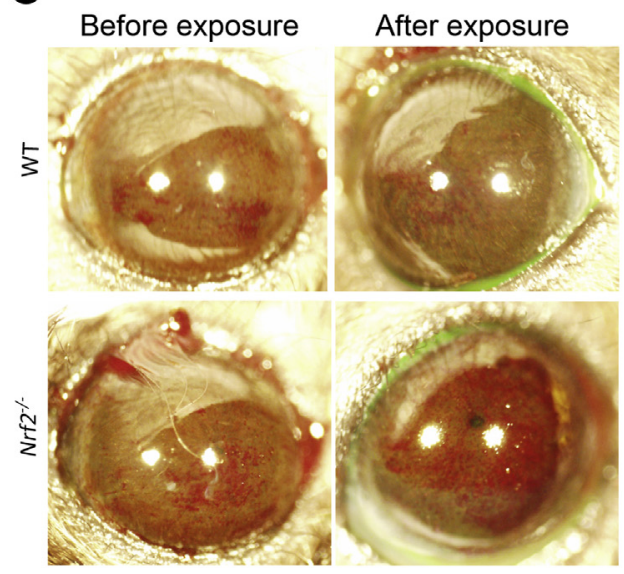

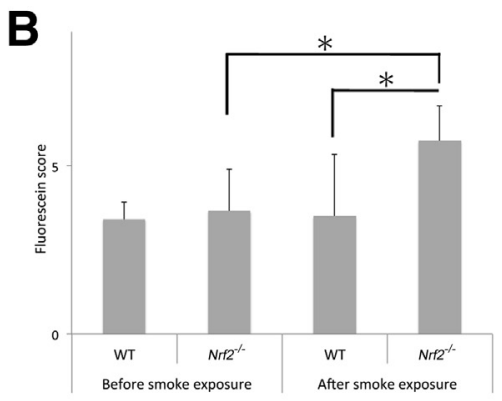

D

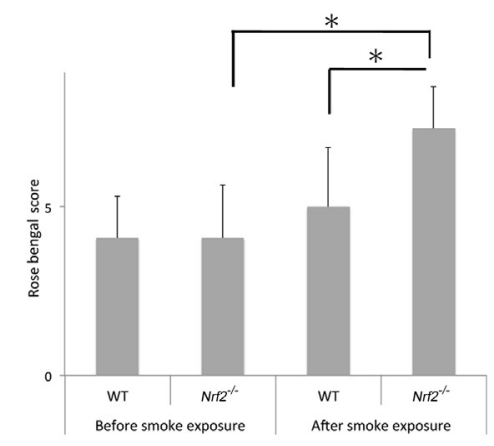

Figure 4 0cular surface damage before and after sidestream cigarette smoke (SCS) exposure in wild-type (WT) and $\mathrm{Nrf2}^{-1-}$ mice. A: Representative fluorescein staining images. B: Fluorescein staining scores in $\mathrm{Nrf2}^{-/-}$mice significantly deteriorated after SCS exposure. Fluorescein staining scores in $\mathrm{Nrf2}^{-/-}$mice after SCS exposure were significantly higher than those in WT mice. C: Representative Rose Bengal staining images. D: Rose Bengal scores Nrf2 ${ }^{-1-}$ mice were significantly worse after SCS exposure. Rose Bengal scores in $\mathrm{Nrf2} 2^{-1-}$ mice after SCS exposure were significantly higher than those in WT mice. Data are given as means \pm SD. ${ }^{*} P<0.05$. mice (Figure 4A). Mean fluorescein staining scores after SCS exposure in $\mathrm{Nrf2^{-/- }}$ mice were higher than those before exposure $(P=0.016)$. After SCS exposure, fluorescein staining scores in $\mathrm{Nrf2^{-/- }}$ mice were significantly higher than those in WT mice $(P=0.008)$ (Figure $4 \mathrm{~B})$. There was no significant difference in Rose Bengal staining scores between mean before and after SCS exposure scores in WT mice (Figure 4C). The mean Rose Bengal score after SCS exposure in $\mathrm{Nrf2^{-/- }}$ mice was higher than the preexposure score $(P<0.001)$. After SCS exposure, Rose Bengal scores in $\mathrm{Nrf2^{-/- }}$ mice were significantly higher than those in WT mice $(P=0.005)$ (Figure $4 \mathrm{D})$.

\section{Lack of Nrf2 Accelerates Oxidative Lipid and DNA} Damage in the Corneal and Conjunctival Epithelia after SCS Exposure

Aldehyde molecules generated endogenously during the process of lipid peroxidation have been reported to be associated with oxidative stress in cells and tissues. ${ }^{14} 4$-HNE is one of the best recognized and most studied cytotoxic products of lipid peroxidation. ${ }^{14}$

To evaluate the influence of the Nrf2 knockout on the lipid peroxidation process, we initially performed corneal and conjunctival staining with anti-4-HNE antibodies (Figure 5, A and B). Corneal and conjunctival epithelia from $\mathrm{Nrf} 2^{-/-}$mice exclusively showed dense staining compared with specimens from WT mice after SCS exposure (Figure 5, A and B).

8-OHdG is a well-known marker for oxidative stress-induced DNA damage. ${ }^{14}$ To assess the cellular DNA damage, we next performed immunohistochemical analysis with anti-8-OHdG antibodies. Nuclei in the cornea and conjunctiva showed scanty staining with $8-\mathrm{OHdG}$ antibodies in WT mice before and after SCS exposure. Similarly, scanty staining was observed in $\mathrm{Nrf} 2^{-/-}$mice before SCS exposure. However, there was a marked increase in nuclear staining in $N r f 2^{-/-}$mice after SCS exposure in the corneal and conjunctival epithelia (Figure 5, C and D).

\section{Decreased Immunohistochemical Staining of Ocular} Surface Mucins in $\mathrm{Nrf2}^{-/}$Mice after SCS Exposure

WT and $N r f 2^{-1-}$ mice showed immunohistochemical membrane-tethered Muc1 mucin expression in the surface of corneal epithelium before SCS exposure. WT mice showed slightly decreased staining of Muc1 in the corneal epithelium after SCS exposure. In contrast, markedly decreased staining of Muc1 was observed in the corneal epithelium of $\mathrm{Nrf2^{-/ }}$ mice after SCS exposure (Figure 6A).

The conjunctival epithelium was abundantly stained with anti-Muc1 antibodies in WT and $\mathrm{Nrf}^{-/-}$mice before SCS exposure. WT mice showed no significant difference in staining pattern after SCS exposure. However, $N r f 2^{-/-}$ mice showed markedly decreased staining of Muc1 in the conjunctival epithelium after SCS exposure (Figure 6B).

The forniceal area of the conjunctival epithelium was also abundantly stained with anti-Muc5ac antibodies in WT and $\mathrm{Nrf}^{-/-}$mice. In WT mice, Muc5ac staining remained unchanged after SCS exposure. In contrast, $\mathrm{Nrf}^{-2^{--}}$mice 

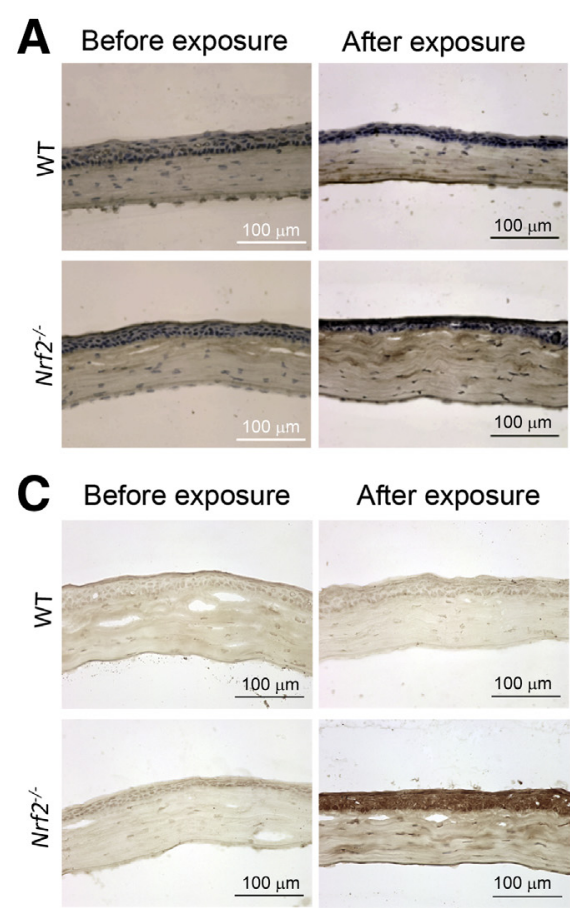

After exposure

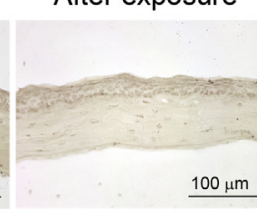

\section{D}
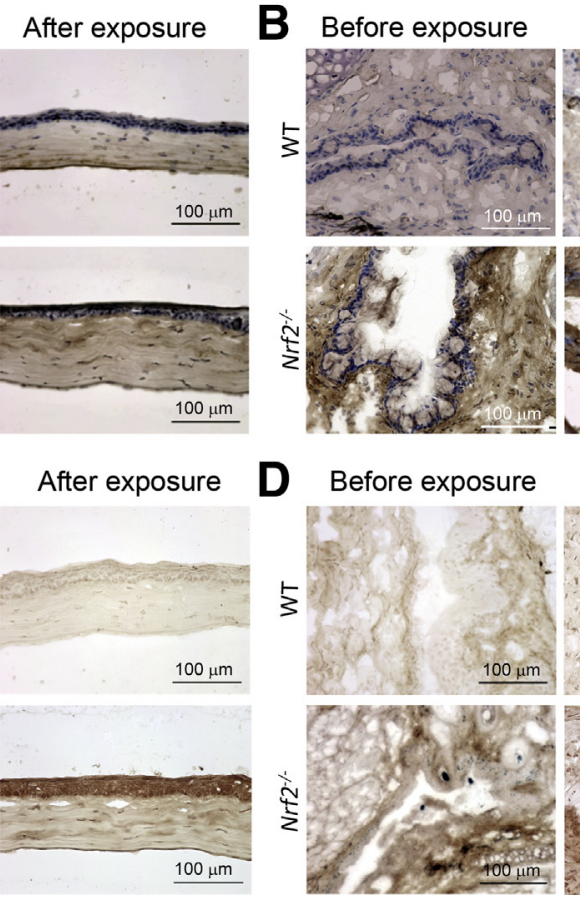

showed a marked decrease in staining of Muc5ac in the conjunctiva after SCS exposure (Figure 6C).

\section{Evaluation of Ocular Surface Mucin and Spdef mRNA Expression in the Corneal and Conjunctival Tissues of $\mathrm{Nrf2}^{-/-}$and WT Mice}

Corneal and conjunctival mRNA expression levels of membrane-tethered Muc1 and Muc4 mucin in $\mathrm{Nrf}^{-/-}$mice significantly decreased after SCS exposure (Figure 7, A-C). Corneal mRNA expression levels of Muc1 after SCS exposure in $\mathrm{Nrf}^{-1-}$ mice were significantly lower than those in WT mice (Figure 7, A and B). Similarly, corneal mRNA expression levels of Muc4 after SCS exposure in $\mathrm{Nrf}^{-/-}$ mice were significantly lower than those in WT mice (Figure 7C). However, conjunctival Muc4 mRNA expression levels did not show differences between WT and $N r f 2^{-/-}$ mice after SCS exposure (Figure 7D). The expression of Muc5ac, which is a gel-forming mucin, in $\mathrm{Nrf} 2^{-/-}$mice significantly decreased after SCS exposure. Muc5ac mRNA expression levels in WT mice after SCS exposure were significantly higher than those in $\mathrm{Nrf}^{-/-}$mice before SCS exposure (Figure 7E). Expression of Spdef, which regulates goblet cell differentiation, was significantly decreased after SCS exposure (Figure 7F). Spdef mRNA expression in $\mathrm{Nrf2^{- \prime - }}$ mice after SCS exposure was significantly lower than that in WT mice after SCS exposure (Figure 7F).

\section{Elevated Inflammatory Cytokine Levels in $\mathrm{Nrf2}^{-/-}$Mice after SCS Exposure}

To investigate the inflammatory cytokine alterations in the tears, we performed cytometric bead array evaluating the

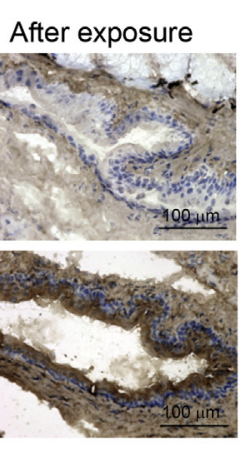

After exposure

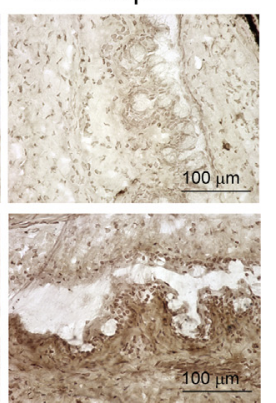

Figure 5 0xidative stress marker immunohistochemical staining in the cornea and conjunctiva before and after sidestream cigarette smoke (SCS) exposure. A and B: Corneal and conjunctival specimens were stained with anti-4-hydroxy-2-nonenal antibody, a lipid oxidative stress marker. After SCS exposure, $\mathrm{Nrf2}^{-/-}$mice showed marked staining in the corneal and conjunctival epithelia. $\mathbf{C}$ and $\mathbf{D}$ : Oxidative DNA damage was also investigated by anti8-hydroxy-2'-deoxyguanosine antibody. C: After SCS exposure, $\mathrm{Nrf2} 2^{-/-}$mice showed marked positive staining in the nucleus of corneal and conjunctival epithelial cells. D: 0 n the other hand, WT mice showed slight increased staining in the conjunctival epithelium after SCS exposure. changes in six cytokines, including IL-1, IL-2, IL-6, IL-10, interferon- $\gamma$, and tumor necrosis factor- $\alpha$. Among them, the mean \pm SD tear IL- 6 concentration in $\mathrm{Nrf}^{-/-}$mice showed a significant increase after SCS exposure (from $0.90 \pm 0.48 \mathrm{pg}$ / $\mathrm{mL}$ to $2.04 \pm 0.78 \mathrm{pg} / \mathrm{mL} ; P=0.013$ ) (Figure $7 \mathrm{G}$ ).

The mean tear IL-10, interferon- $\gamma$, monocyte chemoattractant protein-1, and IL-12p70 levels did not show significant differences before versus after SCS exposure in $N r f 2^{-/-}$ and WT mice (data not shown). There were also no significant differences in the mean IL-10, interferon- $\gamma$, monocyte chemoattractant protein-1, and IL-12p70 concentrations between WT and Sodl ${ }^{-\prime-}$ mice after SCS exposure (data not shown).

\section{Discussion}

Nrf2 plays an important role in sensing oxidative stress and in orchestrating the transcriptional responses to relieve the stressing conditions. ${ }^{15} \mathrm{Nrf} 2$ also plays an important role in cellular protection against chemical materials. ${ }^{16} \mathrm{Nrf} 2$ is widely expressed in tissues with frequent exposure to electrophilic stressors, including lung and gastrointestinal tract. ${ }^{17,18} \mathrm{Nrf} 2^{-/-}$ mice have been reported to be susceptible to tissue injury due to numerous chemical materials and oxidants, including acetaminophen, ${ }^{19,20}$ diesel exhaust particles, ${ }^{21}$ lipopolysaccharide, ${ }^{22}$ and cigarette smoke. ${ }^{23}$

Herein, we found that Nrf2 was highly expressed in the corneal and conjunctival epithelia, which are the first tissue layers exposed to light and/or environmental stress.

SCS exposure of 3 hours for 5 days increased ocular surface damage in $\mathrm{Nrf}^{-/-}$mice. In relation to tear functions, tear volume did not change after SCS exposure. However, tear film stability, measured by breakup time, deteriorated after SCS exposure. From these results, we presumed that 
increased ocular surface damage after SCS exposure may be due to worsening of tear film stability. Previous literature showed that long-term smoking affected tear volume. ${ }^{4,24}$ However, another study reported that acute brief exposure to tobacco smoke did not affect tear volume as measured by the Schirmer test in humans, which were consistent with the present findings. ${ }^{8}$ We speculate that long-term smoking might affect the lacrimal gland systemically, with a

\section{A Before exposure}
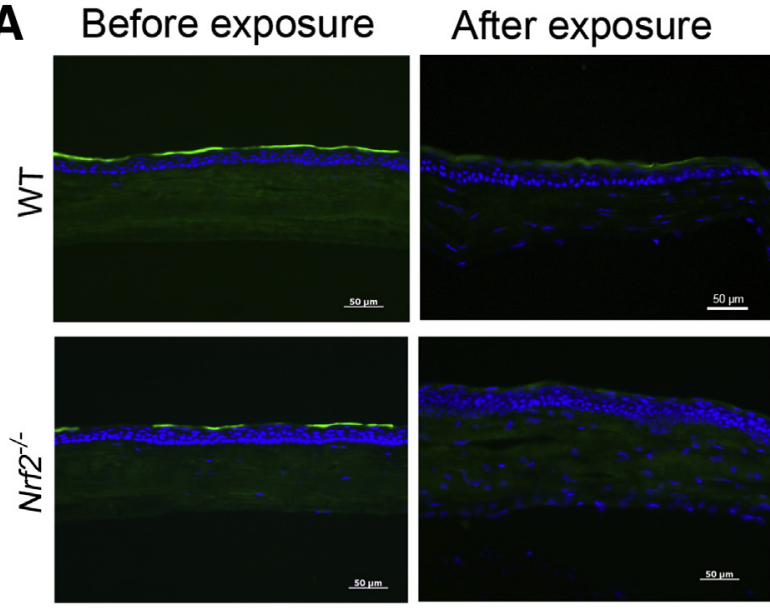

\section{B Before exposure}
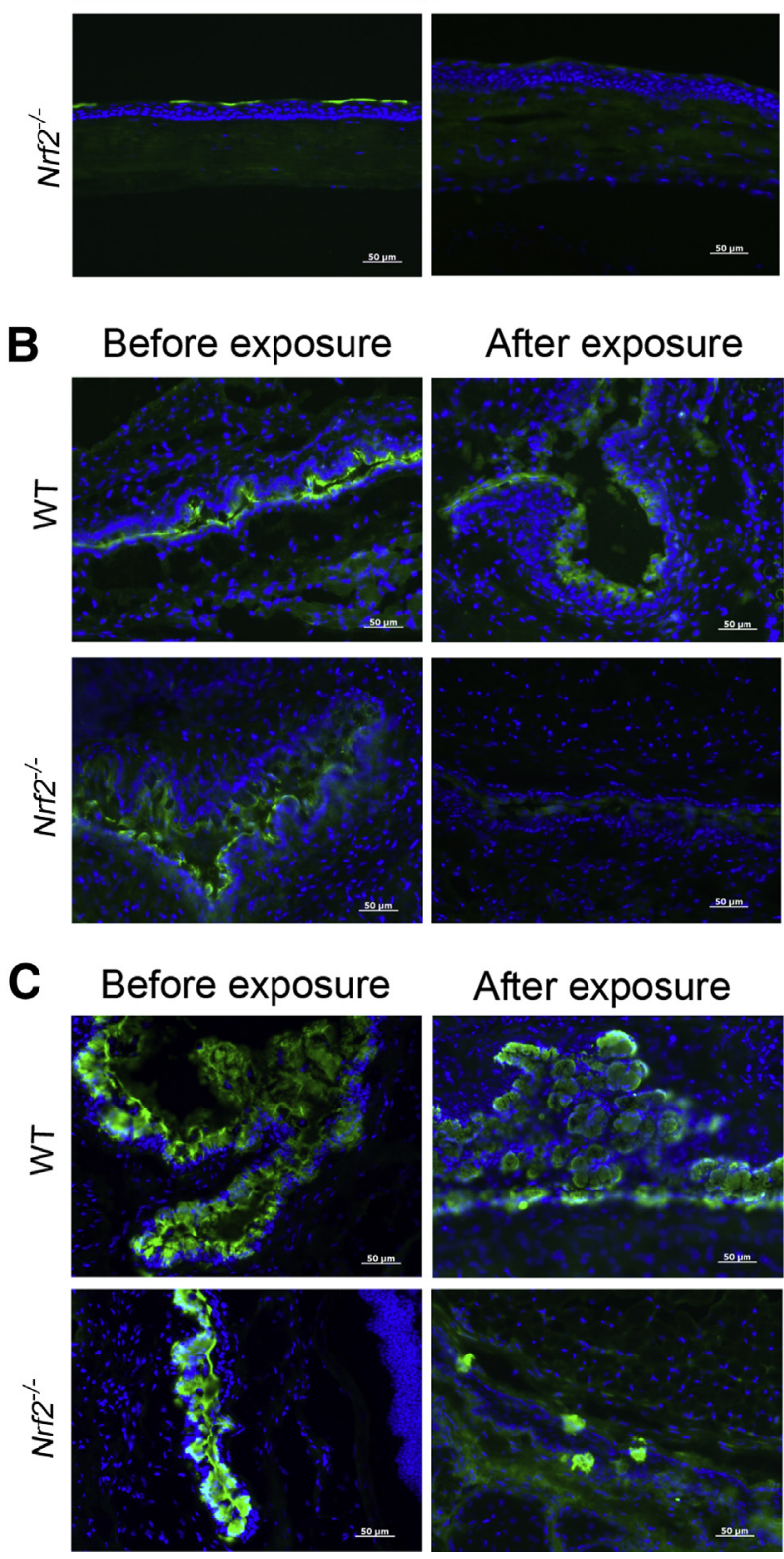

subsequent decrease in tear volume in long-term smokers. Further studies are needed to clarify the differences in tear volume and ocular surface changes between long-term exposure to smoke and brief acute exposure to smoke.

Herein, we did not find any differences in ocular vital staining scores in WT mice before and after SCS exposure. Although the Rose Bengal staining scores in WT mice showed a tendency to increase after cigarette smoke exposure, there were no statistically significant differences. Another study in humans showed that long-term smoking increased ocular surface vital staining scores. ${ }^{25}$ In previous human studies, ${ }^{8,10}$ worsening of vital staining scores was reported even after passive cigarette smoke exposure. In these studies, a constant number of puffs were blown into a controlled smoke chamber, but the accurate amount of cigarette smoke was not monitored. In the present study, a lit cigarette was placed in the chamber to release the SCS. The discrepancies between these studies and the present study might be due to the amount of cigarette smoke exposure. Another reason for such discrepancies may be that the present study used SCS exposure, whereas previous studies used mainstream cigarette smoke exposure. . $^{810}$

Immunohistochemical analysis using antioxidative stress markers 4-HNE and 8-OHdG in the conjunctiva and cornea of $N r 2^{-1-}$ mice showed dense staining compared with WT mice after SCS exposure; however, the extent of staining was similar between the two strains before SCS exposure. Previous studies showed that accumulated oxidative stress induced tissue damage in numerous organs. ${ }^{26-31} \mathrm{Nrf} 2$ plays an important role in protecting tissues against oxidative stress by up-regulating antioxidant enzyme gene on stressed condition. ${ }^{32,33} \mathrm{Nrf}^{-/-}$mice were known to be susceptible to accumulation of oxidative stress. ${ }^{34}$ Defects in Nrf2 may have caused the accumulation of oxidative stress in conjunctival or corneal tissues in $\mathrm{Nrf}^{-/-}$mice after SCS exposure.

$\mathrm{Nrf}^{-/-}$mice also showed scanty staining with Muc1 and Muc5ac after SCS exposure compared with WT mice. Similarly, Muc1 and Muc5ac mRNA expression levels in $N r 2^{-1-}$ mice were significantly lower than those in WT

\footnotetext{
Figure 6 Immunohistochemical staining for ocular surface mucins before and after sidestream cigarette smoke (SCS) exposure in wild-type (WT) and $\mathrm{Nrf2}^{-1-}$ mice. A and B: To evaluate the membrane-tethered mucins, mucin 1 (Muc1) immunohistochemical analysis using an antiMuc1 antibody was performed. The corneal and conjunctival tissues in WT and $\mathrm{Nrf2}^{-/-}$mice showed a continuous positive staining pattern before $\mathrm{SCS}$ exposure in the superficial corneal and conjunctival epithelia. After SCS exposure, WT mice also showed similar immunohistochemical expression. On the other hand, $\mathrm{Nrf2}^{-/-}$mice showed marked scanty staining in the corneal and conjunctival epithelia after SCS exposure. C: To evaluate the secreted tethered mucins, Muc5ac immunohistochemical analysis using an anti-Muc5ac antibody was performed. The conjunctival tissue in WT and $\mathrm{Nrf2}^{-1-}$ mice showed abundant positive-staining cells before SCS exposure in the conjunctival epithelium. After SCS exposure, WT mice also showed similar immunohistochemical expression. On the other hand, $\mathrm{Nrf2}^{-/-}$mice showed a decrease in positive-staining cells in the conjunctival epithelium after SCS exposure.
} 
A

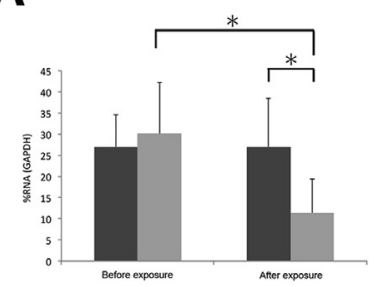

C

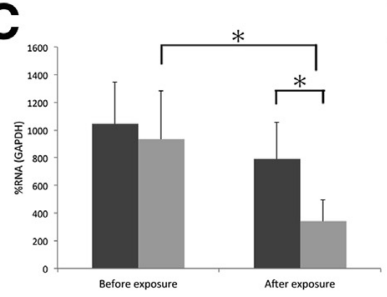

E

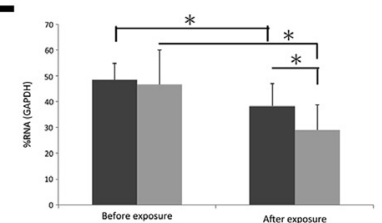

G

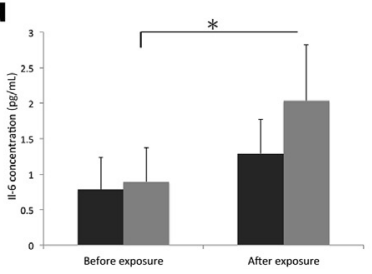

B

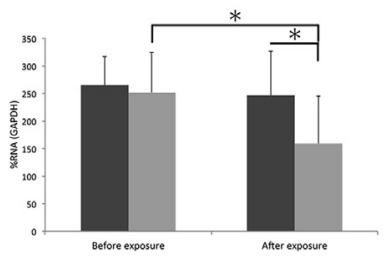

D

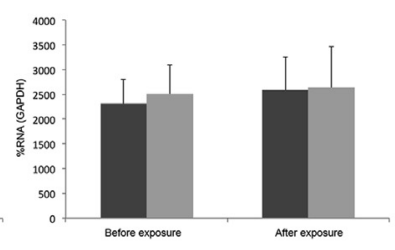

$\mathbf{F}$

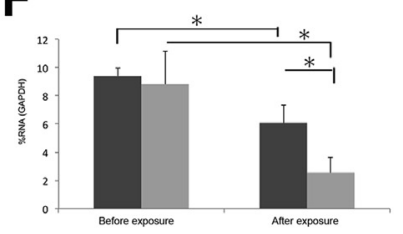

Figure 7 Real-time RT-PCR evaluations of ocular surface mucins and tear cytokine concentrations before and after sidestream cigarette smoke (SCS) exposure. A and B: Conjunctival (A) and corneal Muc1 (B) expression in $\mathrm{Nrf2}^{-1-}$ mice showed a significant decrease in Muc1 expression after SCS exposure. Conjunctival and corneal Muc1 expression in $\mathrm{Nrf2}^{-/-}$mice (light gray bars) was significantly lower than that in wild-type (WT) mice (dark gray bars) after SCS exposure. C: Corneal Muc4 expression in $\mathrm{Nrf2}^{-/-}$mice showed a significant decrease after SCS exposure. D: Conjunctival Muc4 expression in WT and $\mathrm{Nrf2}^{-/-}$mice showed no significant difference after SCS exposure. E: Conjunctival Muc5ac expression in WT and $\mathrm{Nrf2}^{-/-}$mice showed a significant decrease after SCS exposure. Conjunctival Muc5ac expression in $\mathrm{Nrf2}^{-1-}$ mice was significantly lower than that in wild-type mice after SCS exposure. F: Conjunctival SAM-pointed domain epithelial-specific transcription factor (Spdef) expression in wild-type and $\mathrm{Nrf2}^{-/-}$mice showed a significant decrease after SCS exposure. Conjunctival Spdef expression in $\mathrm{Nrf2}^{-/-}$mice was significantly lower than that in WT mice after SCS exposure. G: The mean tear IL-6 concentration in Nrf2 $2^{-1-}$ mice showed a significant increase after $\mathrm{SCS}$ exposure. Data are given as means $\pm \mathrm{SD}$. ${ }^{*} P<0.05$. GAPDH, glyceraldehyde-3-phosphate dehydrogenase.

mice. However, there were no significant differences in conjunctival Muc4 mRNA expression after SCS exposure. A study using a human conjunctival epithelial cell line showed that there were differences in regulation between Muc1 and Muc4 expression after applying serum or retinoic acid. ${ }^{35}$ Although the detailed regulation mechanisms of ocular surface mucins are not well-known, the response to stress conditions, such as SCS exposure, may be different among ocular surface mucins.
The differentiation into goblet cell in the mouse tracheobronchial and gastrointestinal epithelium is highly regulated by Spdef. Deletion of the mouse Spdef gene results in the absence of goblet cells in tracheal/laryngeal submucosal glands and in the conducting airway epithelium after pulmonary allergen exposure in vivo. ${ }^{36} \mathrm{~A}$ recent study showed that Spdef is also required for conjunctival goblet cell differentiation and that $S_{p d e f^{-1}}$ mice have significantly increased corneal surface fluorescein staining, a phenotype consistent with dry eye. ${ }^{37}$ After SCS exposure, we also found notable change regarding Spdef expression in conjunctival tissue. In WT and $N r f 2^{-/-}$mice, Spdef expression decreased after SCS exposure. Moreover, Spdef expression in $\mathrm{Nrf}^{-/-}$mice was lower than that in WT mice after SCS exposure.

Previous literature proved that cigarette smoke exposure induced histologic changes in the upper and lower airways. ${ }^{38}$ Hyperplasia and squamous metaplasia of the respiratory epithelium of the dorsal nasal turbinates was reported in rats after SCS exposure. ${ }^{39}$

After SCS exposure, accumulation of oxidative stress in the ocular surface may have altered the phenotype of conjunctival epithelium and decreased mucin expression in the conjunctival epithelium. We believe that these changes deteriorated the tear film stability and then led to ocular surface damage.

Although the relation between oxidative stress and differentiation of the conjunctival epithelium is still unknown, the present study suggested the possibility that Nrf2 is associated with differentiation of the conjunctival epithelium. Goblet cells secrete mucin and are essential for the maintenance of tear film stability. ${ }^{37}$ Goblet cell densities decrease in various types of dry eyes, including Sjögren syndrome, Stevens-Johnson syndrome, and graft-versushost disease. ${ }^{40-42}$

Recently, we reported the accumulation of lipid oxidative stress in tear and conjunctival specimens in Sjögren syndrome patients. ${ }^{43}$ The conjunctiva in Sjögren syndrome patients was reported to show squamous metaplasia accompanied by the up-regulation of pro-keratinization genes, such as transglutaminase- 1 and involucrin. ${ }^{44}$

Regulation of Muc5ac expression by Nrf2 was previously investigated using human airway epithelial cells. ${ }^{45}$ This study showed that in human airway epithelial cells, Nrf2 gene expression was repressed using siRNA, showing accumulation of reactive oxygen species and up-regulation of Muc5ac gene expression. The present study was conducted by using an in vivo SCS exposure system that involved surrounding factors, such as the tear film and mechanical friction from the eyelids, hence the conflict between these results.

There were some limitations in this study. Only male mice were analyzed to avoid the effect of menstrual cycle in the study. Because dry eye is more prevalent in women, we need to investigate the phenotypic alterations of the ocular surface with SCS exposure using female mice in the future.

A previous human study showed that brief passive smoke exposure increased the tear lipid oxidative stress marker and 
IL-6 concentrations after brief mainstream cigarette smoke exposure. $^{8}$ The present study also showed that tear IL-6 concentrations increased in $\mathrm{Nrf}^{-/-}$mice after SCS exposure. On the other hand, a previous study using a rat cigarette smoke exposure model showed that brief passive smoke exposure induced up-regulation of cytochrome P450s in lung and liver. ${ }^{9}$ Based on these findings, we speculated that ocular surface changes after SCS exposure resulted from systemic and direct local effects from the SCS. The direct diffusion of toxic materials or oxidants could alter the ocular surface epithelium. The systemic accumulation of oxidants or chemical compounds from cigarette smoke could also affect the lacrimal gland and change the quality or stability of tear fluid. Further studies to clarify the detailed pathogenesis of ocular surface changes after SCS exposure should be investigated in future studies.

In conclusion, $N r 2^{-/-}$mice showed decreased ocular surface mucin expression and excessive accumulation of oxidative stress concomitant with tear instability and ocular surface abnormalities after SCS exposure. It is possible that Nrf2 plays an important role in protection of the ocular surface against cigarette smoke.

\section{Acknowledgment}

We thank Prof. Masayuki Yamamoto (Tohoku University Graduate School of Medicine, Sendai, Japan) for generously providing $\mathrm{Nrf}^{-/-}$mice.

\section{References}

1. Meltzer EO: Prevalence, economic, and medical impact of tobacco smoking. Ann Allergy 1994, 73:381-389. quiz 8-91

2. Lofroth G: Environmental tobacco smoke: overview of chemical composition and genotoxic components. Mutat Res 1989, 222:73-80

3. Takahashi A, Iwasaki I, Ide G: Effects of minute amounts of cigarette smoke with or without nebulized N-nitroso-N-methylurethane on the respiratory tract of mice. Jpn J Cancer Res 1985, 76:324-330

4. Matsumoto Y, Dogru M, Goto E, Sasaki Y, Inoue H, Saito I, Shimazaki J, Tsubota K: Alterations of the tear film and ocular surface health in chronic smokers. Eye (Lond) 2008, 22:961-968

5. Weber A: Annoyance and irritation by passive smoking. Prev Med 1984, 13:618-625

6. Moss SE, Klein R, Klein BE: Prevalence of and risk factors for dry eye syndrome. Arch Ophthalmol 2000, 118:1264-1268

7. Chia EM, Mitchell P, Rochtchina E, Lee AJ, Maroun R, Wang JJ: Prevalence and associations of dry eye syndrome in an older population: the Blue Mountains Eye Study. Clin Experiment Ophthalmol 2003, 31:229-232

8. Rummenie VT, Matsumoto Y, Dogru M, Wang Y, Hu Y, Ward SK, Igarashi A, Wakamatsu T, Ibrahim O, Goto E, Luyten G, Inoue H, Saito I, Shimazaki J, Tsubota K: Tear cytokine and ocular surface alterations following brief passive cigarette smoke exposure. Cytokine 2008, 43:200-208

9. Higuchi A, Ito K, Dogru M, Kitamura M, Mitani F, Kawakita T, Ogawa Y, Tsubota K: Corneal damage and lacrimal gland dysfunction in a smoking rat model. Free Radic Biol Med 2011, 51:2210-2216

10. Ward SK, Dogru M, Wakamatsu T, Ibrahim O, Matsumoto $\mathrm{Y}$, Kojima T, Sato EA, Ogawa J, Schnider C, Negishi K, Tsubota K:
Passive cigarette smoke exposure and soft contact lens wear. Optom Vis Sci 2010, 87:367-372

11. Motohashi H, Yamamoto M: Nrf2-Keap1 defines a physiologically important stress response mechanism. Trends Mol Med 2004, 10: $549-557$

12. Banning A, Deubel S, Kluth D, Zhou Z, Brigelius-Flohe R: The GIGPx gene is a target for Nrf2. Mol Cell Biol 2005, 25:4914-4923

13. Dursun D, Wang M, Monroy D, Li DQ, Lokeshwar BL, Stern ME, Pflugfelder SC: A mouse model of keratoconjunctivitis sicca. Invest Ophthalmol Vis Sci 2002, 43:632-638

14. Dalle-Donne I, Rossi R, Colombo R, Giustarini D, Milzani A: Biomarkers of oxidative damage in human disease. Clin Chem 2006, 52 : $601-623$

15. Knorr-Wittmann C, Hengstermann A, Gebel S, Alam J, Muller T: Characterization of Nrf2 activation and heme oxygenase-1 expression in NIH3T3 cells exposed to aqueous extracts of cigarette smoke. Free Radic Biol Med 2005, 39:1438-1448

16. Taguchi K, Maher JM, Suzuki T, Kawatani Y, Motohashi H, Yamamoto M: Genetic analysis of cytoprotective functions supported by graded expression of Keap1. Mol Cell Biol 2010, 30:3016-3026

17. Chan K, Lu R, Chang JC, Kan YW: NRF2, a member of the NFE2 family of transcription factors, is not essential for murine erythropoiesis, growth, and development. Proc Natl Acad Sci U S A 1996, 93 : 13943-13948

18. Itoh $\mathrm{K}$, Chiba $\mathrm{T}$, Takahashi $\mathrm{S}$, Ishii $\mathrm{T}$, Igarashi $\mathrm{K}$, Katoh $\mathrm{Y}$, Oyake $\mathrm{T}$, Hayashi N, Satoh K, Hatayama I, Yamamoto M, Nabeshima Y: An Nrf2/small Maf heterodimer mediates the induction of phase II detoxifying enzyme genes through antioxidant response elements. Biochem Biophys Res Commun 1997, 236:313-322

19. Chan K, Han XD, Kan YW: An important function of Nrf2 in combating oxidative stress: detoxification of acetaminophen. Proc Natl Acad Sci U S A 2001, 98:4611-4616

20. Enomoto A, Itoh K, Nagayoshi E, Haruta J, Kimura T, O'Connor T, Harada T, Yamamoto M: High sensitivity of Nrf2 knockout mice to acetaminophen hepatotoxicity associated with decreased expression of ARE-regulated drug metabolizing enzymes and antioxidant genes. Toxicol Sci 2001, 59:169-177

21. Aoki Y, Sato H, Nishimura N, Takahashi S, Itoh K, Yamamoto M: Accelerated DNA adduct formation in the lung of the Nrf2 knockout mouse exposed to diesel exhaust. Toxicol Appl Pharmacol 2001, 173: $154-160$

22. Thimmulappa RK, Scollick C, Traore K, Yates M, Trush MA, Liby KT, Sporn MB, Yamamoto M, Kensler TW, Biswal S: Nrf2dependent protection from LPS induced inflammatory response and mortality by CDDO-Imidazolide. Biochem Biophys Res Commun 2006, 351:883-889

23. Iizuka $\mathrm{T}$, Ishii $\mathrm{Y}$, Itoh $\mathrm{K}$, Kiwamoto $\mathrm{T}$, Kimura $\mathrm{T}$, Matsuno $\mathrm{Y}$, Morishima Y, Hegab AE, Homma S, Nomura A, Sakamoto T, Shimura M, Yoshida A, Yamamoto M, Sekizawa K: Nrf2-deficient mice are highly susceptible to cigarette smoke-induced emphysema. Genes Cells 2005, 10:1113-1125

24. Sayin N, Kara N, Pekel G, Altinkaynak H: Effects of chronic smoking on central corneal thickness, endothelial cell, and dry eye parameters. Cutan Ocul Toxicol 2014, 33:201-205

25. Thomas J, Jacob GP, Abraham L, Noushad B: The effect of smoking on the ocular surface and the precorneal tear film. Australas Med J 2012, 5:221-226

26. Kojima T, Wakamatsu TH, Dogru M, Ogawa Y, Igarashi A, Ibrahim OM, Inaba T, Shimizu T, Noda S, Obata H, Nakamura S, Wakamatsu A, Shirasawa T, Shimazaki J, Negishi K, Tsubota K: Agerelated dysfunction of the lacrimal gland and oxidative stress: evidence from the $\mathrm{Cu}, \mathrm{Zn}$-superoxide dismutase-1 (Sod1) knockout mice. Am J Pathol 2012, 180:1879-1896

27. Yuki K, Ozawa Y, Yoshida T, Kurihara T, Hirasawa M, Ozeki N, Shiba D, Noda K, Ishida S, Tsubota K: Retinal ganglion cell loss in superoxide dismutase 1 deficiency. Invest Ophthalmol Vis Sci 2011, 52:4143-4150 
28. Schapira AH, Tolosa E: Molecular and clinical prodrome of Parkinson disease: implications for treatment. Nat Rev Neurol 2010, 6:309-317

29. Higgins GC, Beart PM, Shin YS, Chen MJ, Cheung NS, Nagley P: Oxidative stress: emerging mitochondrial and cellular themes and variations in neuronal injury. J Alzheimers Dis 2010, 20(Suppl 2): S453-S473

30. Reddy PH: Amyloid precursor protein-mediated free radicals and oxidative damage: implications for the development and progression of Alzheimer's disease. J Neurochem 2006, 96:1-13

31. Milei J, Forcada P, Fraga CG, Grana DR, Iannelli G, Chiariello M, Tritto I, Ambrosio G: Relationship between oxidative stress, lipid peroxidation, and ultrastructural damage in patients with coronary artery disease undergoing cardioplegic arrest/reperfusion. Cardiovasc Res 2007, 73:710-719

32. Himori N, Yamamoto K, Maruyama K, Ryu M, Taguchi K, Yamamoto M, Nakazawa T: Critical role of Nrf2 in oxidative stressinduced retinal ganglion cell death. J Neurochem 2013, 127:669-680

33. Yagishita Y, Fukutomi T, Sugawara A, Kawamura H, Takahashi T, Pi J, Uruno A, Yamamoto M: Nrf2 protects pancreatic beta-cells from oxidative and nitrosative stress in diabetic model mice. Diabetes 2014, 63:605-618

34. Hyeon S, Lee H, Yang Y, Jeong W: Nrf2 deficiency induces oxidative stress and promotes RANKL-induced osteoclast differentiation. Free Radic Biol Med 2013, 65:789-799

35. Hori Y, Spurr-Michaud S, Russo CL, Argueso P, Gipson IK: Differential regulation of membrane-associated mucins in the human ocular surface epithelium. Invest Ophthalmol Vis Sci 2004, 45:114-122

36. Chen G, Korfhagen TR, Xu Y, Kitzmiller J, Wert SE, Maeda Y, Gregorieff A, Clevers H, Whitsett JA: SPDEF is required for mouse pulmonary goblet cell differentiation and regulates a network of genes associated with mucus production. J Clin Invest 2009, 119:2914-2924
37. Marko CK, Menon BB, Chen G, Whitsett JA, Clevers H, Gipson IK: Spdef null mice lack conjunctival goblet cells and provide a model of dry eye. Am J Pathol 2013, 183:35-48

38. Dye JA, Adler KB: Effects of cigarette smoke on epithelial cells of the respiratory tract. Thorax 1994, 49:825-834

39. von Meyerinck L, Scherer G, Adlkofer F, Wenzel-Hartung R, Brune H, Thomas C: Exposure of rats and hamsters to sidestream smoke from cigarettes in a subchronic inhalation study. Exp Pathol 1989, 37:186-189

40. Gipson IK, Hori Y, Argueso P: Character of ocular surface mucins and their alteration in dry eye disease. Ocul Surf 2004, 2:131-148

41. Nelson JD, Wright JC: Conjunctival goblet cell densities in ocular surface disease. Arch Ophthalmol 1984, 102:1049-1051

42. Wang Y, Ogawa Y, Dogru M, Tatematsu Y, Uchino M, Kamoi M, Okada N, Okamoto S, Tsubota K: Baseline profiles of ocular surface and tear dynamics after allogeneic hematopoietic stem cell transplantation in patients with or without chronic GVHD-related dry eye. Bone Marrow Transplant 2010, 45:1077-1083

43. Wakamatsu TH, Dogru M, Matsumoto Y, Kojima T, Kaido M, Ibrahim OM, Sato EA, Igarashi A, Ichihashi Y, Satake Y, Shimazaki J, Tsubota K: Evaluation of lipid oxidative stress status in Sjogren syndrome patients. Invest Ophthalmol Vis Sci 2013, 54: $201-210$

44. Hirai N, Kawasaki S, Tanioka H, Connon CJ, Yamasaki K, Yokoi N, Komuro A, Kinoshita S: Pathological keratinisation in the conjunctival epithelium of Sjogren's syndrome. Exp Eye Res 2006, 82: $371-378$

45. Qi L, Xiangdong Z, Hongmei Y, Xiaohong N, Xiaoyan X: Regulation of neutrophil elastase-induced MUC5AC expression by nuclear factor erythroid-2 related factor 2 in human airway epithelial cells. J Investig Med 2010, 58:730-736 